Check for updates

Cite this: RSC Adv., 2019, 9, 30815

Received 21st August 2019

Accepted 16th September 2019

DOI: 10.1039/c9ra06574a

rsc.li/rsc-advances

\section{A biobased Schiff base from protocatechualdehyde and its application in flame-retardant, low-smoke epoxy resin systems $\dagger$}

\begin{abstract}
Weiqi Xie, (D) a Shiwen Huang, ${ }^{a}$ Shumei Liu (D) *ab and Jianqing Zhao*ab

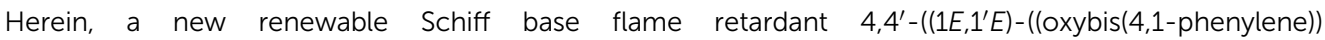
bis(azanylylidene))bis(methanylylidene))bis(benzene-1,2-diol) (PH-ODA) was prepared by the reaction of protocatechualdehyde with 4,4'-diaminodiphenyl ether (ODA). PH-ODA (acting as a carbonization agent) combined with ammonium polyphosphate (APP) were used as intumescent flame retardants for commercial bisphenol A epoxy resin (DGEBA). For the cured epoxy resin containing 7.5\% APP and $2.5 \%$ $\mathrm{PH}-\mathrm{ODA}$, the limiting oxygen index (LOI) reached $29.9 \%$ (with the $\mathrm{V}-0$ rating in UL-94 test), and the peak heat release rate and total smoke production were respectively decreased by $88.1 \%$ and $68.3 \%$, compared with pure epoxy resin. The enhancement of fire-safety performance was due to PH-ODA/APP promoting the formation of a compact intumescent char structure. It was also found that the synergism between PH-ODA and APP was helpful to enhance the fire resistance of the epoxy matrix. This work provides a facile and sustainable route for synthesizing Schiff base compounds from biomass-derived resources, possessing great potential for application in highly-effective intumescent flame retardants.
\end{abstract}

\section{Introduction}

Epoxy resins are a critical class of polymeric materials and have been utilized in various areas (i.e., coatings, adhesives, and laminate materials) for their excellent performance (dimensional stability, satisfactory chemical resistance, good mechanical properties). ${ }^{1-4}$ However, the poor fire safety of epoxy resins is one of the main drawbacks and greatly limits their applications in some fields that require fire resistance..$^{5-7}$

Different types of flame retardants (phosphorus-, nitrogenand silicon-containing compounds, metal hydroxides and intumescent flame retardants (IFRs)) have been adopted for enhancing the fire-safety performance of epoxy resins. ${ }^{8-11}$ Among them, IFRs are eco-friendly flame retardants with many advantages (e.g., low toxicity and smoke). ${ }^{12,13}$ However, the high addition (more than $25 \mathrm{wt} \%$ ) of traditional IFRs (such as ammonium polyphosphate and pentaerythritol) is usually required to achieve the fire-safety properties of epoxy resins. ${ }^{\mathbf{1 4}}$ The high addition of IFRs often caused some new problems like the poor mechanical and chemical compatibility performance

${ }^{a}$ School of Materials Science and Engineering, South China University of Technology, Guangzhou, 510640, P. R. China.E-mail: liusm@scut.edu.cn; psjqzhao@scut.edu. cn; Fax: +86-13611400566; +86-13609724000; Tel: +86-13611400566; +8613609724000

${ }^{b}$ Key Laboratory of Polymer Processing Engineering, Ministry of Education, Guangzhou 510640, P. R. China

$\dagger$ Electronic supplementary information (ESI) available. See DOI: 10.1039/c9ra06574a of the epoxy matrix. ${ }^{15}$ Hence, the fire resistance efficiency of IFRs should be further improved for reducing the adverse impact on other properties of the epoxy thermosets. Recently, many works were reported for solving this problem, and the synthesis of high-performance carbonization agents of IFRs was considered as an important strategy. ${ }^{16-19}$

Schiff base compounds are very important chemicals, which are widely applied for many applications (i.e., drug release, gas separation, and catalysts) because of their diverse properties (i.e., catalytic, magnetic, and biological properties). ${ }^{20-23}$ Recently, the applications of Schiff base compounds for the flame retardant area has attracted much attention due to their good cross-linking charring ability at high temperature. ${ }^{24,25}$ Zhang's work ${ }^{26}$ revealed that the $\mathrm{C}=\mathrm{N}$ double bonds in Schiff base compounds were able to generate nitrogen-containing hexatomic ring at higher temperature. These hexatomic ring structures make polymers form stable cross-linked networks and endow them with high flame retardancy. Obviously, Schiff base compounds have great potential for the carbonization agents of IFRs. Besides, most of the carbonization agents (i.e., pentaerythritol) used in IFRs are produced from the petroleumbased resource. ${ }^{27}$ In order to meet the urgent needs for alleviating the shortage of fossil resources and achieving the sustainable development, the preparation of bio-based Schiff base carbonization agents for IFRs is a potential and sustainable solution. ${ }^{28}$ Protocatechualdehyde is a plant-derived phenolic aldehyde compound containing two phenolic hydroxyl groups and an aldehyde group, which has been widely used in medicine because of its biological activities. ${ }^{29,30}$ Based 
on its unique structures and functional groups, protocatechualdehyde might be a promising biomass resource for preparing highly-effective Schiff base flame retardants.

Herein, a novel biorenewable flame retardant $4,4^{\prime}-\left(\left(1 E, 1^{\prime} E\right)-\right.$ ((oxybis(4,1-phenylene))bis(azanylylidene))

bis(methanylylidene))bis(benzene-1,2-diol) (PH-ODA) is prepared from renewable protocatechualdehyde and employed as the carbonization agent of IFRs for commercial bisphenol A epoxy resin (DGEBA). It is expected that the flame retardant from a novel biomass-derived aromatic Schiff base compound has outstanding fire resistance efficiency. Moreover, the thermomechanical, thermal and mechanical properties of the cured resins are also evaluated.

\section{Materials and methods}

\section{Materials}

Protocatechualdehyde, 4,4'-diaminodiphenyl ether (ODA), ammonium polyphosphate (APP), and 4,4'-methylenedianiline (DDM) were obtained from Aladdin Reagent Co. Ltd., China. Ethanol, ethyl acetate and acetone were obtained from Guangzhou Chemical Reagent Factory, China. DGEBA (epoxy value $=0.51 \mathrm{~mol} / 100 \mathrm{~g}$ ) was purchased from SINOPEC Baling company, China.

\section{Synthesis of Schiff base PH-ODA}

In $\mathrm{N}_{2}$ atmosphere, protocatechualdehyde ( $41.4 \mathrm{~g}, 0.30 \mathrm{~mol}$ ) was dissolved in $350 \mathrm{~mL}$ of ethanol. Then, 4,4'-diaminodiphenyl ether (19.8 g, $0.10 \mathrm{~mol})$ was slowly added into this system. After continuous stirring for $30 \mathrm{~min}$ at room temperature, the system was heated to $80{ }^{\circ} \mathrm{C}$ and stirred for $6 \mathrm{~h}$. Afterward, the mixture was cooled down and transferred to ice water. The crude product was filtered and washed three times by ethyl acetate to give PH-ODA as a yellow powder (39.0 g, yield 88.7\%).

\section{Preparation of cured epoxy thermosets}

The epoxy thermosets were prepared and the formulations of the PH-ODA/APP/DDM/DGEBA system were displayed in Table 1 (the molar ratio of reactive hydrogens to the epoxy groups is 1). Based on the previous works on IFRs system, the selected weight ratio of APP and carbonization agent (PH-ODA) was $3: 1$ for IFRs in this work. ${ }^{31}$ DDM, PH-ODA, and APP were firstly dissolved in acetone $\left(50{ }^{\circ} \mathrm{C}\right)$. Then, the solution was added to

Table 1 Stoichiometric formulation of the epoxy system

\begin{tabular}{lllll}
\hline Samples $^{a}$ & DGEBA (wt\%) & DDM (wt\%) & APP (wt\%) & PH-ODA (wt\%) \\
\hline PH-ODA-0 & 79.83 & 20.17 & 0 & 0 \\
PH-ODA-5 & 75.84 & 19.16 & 3.75 & 1.25 \\
APP-10 & 71.85 & 18.15 & 10 & 0 \\
PH-ODA-10 & 71.85 & 18.15 & 7.5 & 2.5 \\
PH-ODA-15 & 67.86 & 17.14 & 11.25 & 3.75
\end{tabular}

${ }^{a}$ Sample name: PH-ODA- $X, X$ represents the mass fraction of the sum of PH-ODA and APP in curing system. APP-10 represents $10 \mathrm{wt} \%$ of APP in curing system. a beaker containing DGEBA and stirred for $20 \mathrm{~min}\left(50{ }^{\circ} \mathrm{C}\right)$. Afterward, the curing system was transferred in a mould and degassed at $60{ }^{\circ} \mathrm{C}$ for $30 \mathrm{~min}$. Finally, the system was cured at $80{ }^{\circ} \mathrm{C}$ for $2 \mathrm{~h}, 110^{\circ} \mathrm{C}$ for $1 \mathrm{~h}, 150^{\circ} \mathrm{C}$ for $2 \mathrm{~h}$, and $180^{\circ} \mathrm{C}$ for $2 \mathrm{~h}$.

\section{Characterizations}

${ }^{1} \mathrm{H}$ and ${ }^{13} \mathrm{C}$ nuclear magnetic resonance (NMR) spectra were collected with a Bruker NMR spectrometer (Billerica, MA, USA) and deuterated dimethylsulfoxide $\left(\mathrm{DMSO}_{\mathrm{C}} \mathrm{d}_{6}\right)$ was used as the solvent. The infrared spectra (FT-IR) were obtained with a Vertex 70 spectrometer (Bruker, Billerica, MA, USA) using KBr pellets. Mass spectrometry (MS) spectra were collected with a maXis impact mass spectrometer (Bruker).

Thermogravimetric analyses (TGA) was conducted using a TG-209F1 TGA (Netzsch, Selb, Germany) at a heating rate of $10{ }^{\circ} \mathrm{C} \min ^{-1}\left(\mathrm{~N}_{2}\right.$ atmosphere), and the temperature range is from 50 to $700{ }^{\circ} \mathrm{C}$. Dynamic mechanical analysis (DMA) was conducted using a TA instrument (DMA Q800, America) at a heating rate of $3{ }^{\circ} \mathrm{C} \mathrm{min}^{-1}$ (from 25 to $230^{\circ} \mathrm{C}$ ). The dimensions of cured samples for measurement were $40 \times 10 \times 3.0 \mathrm{~mm}$.

Tensile and flexural properties were analyzed based on ASTM D638-08 and ASTM D790-07, respectively, on an Instron-5967 universal electronic testing machine.

UL-94 vertical burning tests were conducted with a UL 94 flame chamber (Fire Testing Technology, UK) according to ASTM D3801-10 (sample dimension of $125 \times 13 \times 3 \mathrm{~mm}$ ). Limiting oxygen index (LOI) tests were conducted using an oxygen index instrument (Fire Testing Technology, UK) according to ASTM D2863-97 (sample dimension of $150 \times 6.5 \times$ $3.2 \mathrm{~mm}$ ). Cone calorimeter tests (CCT) were conducted using a FTT cone calorimeter according to ISO5660 (sample dimension of $100 \times 100 \times 5 \mathrm{~mm})$.

Scanning electron microscopy (SEM) experiments were conducted with a NOVA NANOSEM 430 machine. The sample was sputter-coated with gold before testing. X-ray photoelectron spectrum (XPS) was conducted using an Axis Ultra spectrometer (Kratos, England). Thermogravimetry-Fourier transform infrared spectrometer (TGA-FTIR) tests were conducted with a STA449C/3MFC/G instrument (Bruker, USA) $\left(\mathrm{N}_{2}\right.$ atmosphere, heating rate $=20{ }^{\circ} \mathrm{C} \min ^{-1}$ ).

\section{Results and discussion}

\section{Synthesis of PH-ODA}

The bio-based aromatic Schiff base compound PH-ODA is synthesized from protocatechualdehyde and ODA in one step depicted in Fig. 1. The synthesis method is facile and high-yield, which provides a feasible way for the scalable and sustainable production of bio-based flame retardant. The structure of the bio-based PH-ODA is characterized using ${ }^{1} \mathrm{H}-\mathrm{NMR},{ }^{13} \mathrm{C}-\mathrm{NMR}$ and HRESI-MS techniques (Fig. S1 $\dagger$ ). Fig. S1a $\dagger$ displays the ${ }^{1} \mathrm{H}-\mathrm{NMR}$ spectrum of PH-ODA, and the peak at 9.58 and $9.30 \mathrm{ppm}$ are attributed to $-\mathrm{OH}$. $\left(\mathrm{H}_{1}\right.$ and $\left.\mathrm{H}_{2}\right)$, the peak at $8.39 \mathrm{ppm}$ is attributed to $-\mathrm{CH}=\mathrm{N}\left(\mathrm{H}_{6}\right)$, the signal of the aromatic protons $\left(\mathrm{H}_{3-5}, \mathrm{H}_{7}, \mathrm{H}_{8}\right)$ is found at 7.40-6.83 ppm. In the ${ }^{13} \mathrm{C}-\mathrm{NMR}$ spectrum of PH-ODA (Fig. S1b $\dagger$ ), the expected 


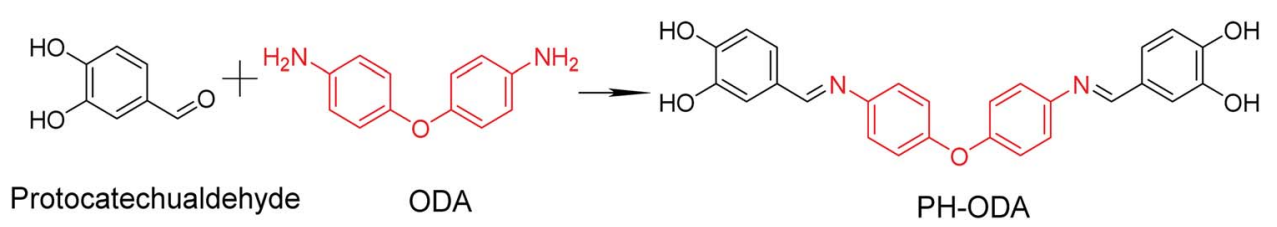

Fig. 1 Synthesis route for $\mathrm{PH}-\mathrm{ODA}$.

chemical shifts of $\mathrm{C}$ atoms are in good agreement with the actual chemical shifts. Fig. S1c $\dagger$ shows an $\left[\mathrm{M}+\mathrm{H}^{+}\right]$ion peak at $m / z 441.14$ (molecular formula $=\mathrm{C}_{26} \mathrm{H}_{20} \mathrm{~N}_{2} \mathrm{O}_{5}$ ). All these results verify that $\mathrm{PH}-\mathrm{ODA}$ with a designed structure has been successfully synthesized. The thermal stability of PH-ODA is assessed by TGA ( $\mathrm{N}_{2}$ atmosphere). As shown in Fig. S1d, $\uparrow$ the initial decomposition temperature $\left(T_{5 \%}\right.$, temperature at $5 \%$ weight loss) of PH-ODA is $256.2{ }^{\circ} \mathrm{C}$, and PH-ODA presents very high residue $(60.1 \%)$ at $700^{\circ} \mathrm{C}$, suggesting an excellent charring ability of PH-ODA.

\section{Flame-retarded properties}

Vertical burning (UL-94) and LOI tests are utilized for evaluating the fire resistance properties (Table 2) ${ }^{32}$ The pure epoxy resin PH-ODA-0 displays the LOI value of $23.5 \%$ with melt-dripping and no rating in UL-94 test. $5.0 \mathrm{wt} \% \mathrm{PH}-\mathrm{ODA} / \mathrm{APP}$ increases the LOI value to $27.8 \%$ without any dripping. By only adding $10 \mathrm{wt} \%$ APP to epoxy resin, the LOI value of sample APP-10 reaches $29.1 \%$ with no rating in UL-94 test. The fire-safety of epoxy resins is further improved by replacing APP with PH-ODA/ APP, and $10.0 \mathrm{wt} \%$ PH-ODA/APP (sample PH-ODA-10) increases LOI value to $29.9 \%$ with $\mathrm{V}-0$ rating of UL-94 test. The above results reveal that PH-ODA/APP exhibits excellent efficiency on flame-retarded performance, and PH-ODA has synergistic effect with APP in enhancing the fire-safety performance.

The fire-safety of the epoxy thermosets is further assessed with cone calorimeter test (CCT) (Fig. 2 and Table 3). ${ }^{33}$ As seen, the time to ignition (TTI) value of PH-ODA-10 is much lower than PH-ODA-0, which is mainly caused by the degradation of IFRs at the early stage. ${ }^{24}$ The peak heat release rate (pHRR) of PH-ODA-10 is decreased to $124.3 \mathrm{~kW} \mathrm{~m}^{2}$, which is $88.1 \%$ lower than PH-ODA-0 (1045.2 $\left.\mathrm{kW} \mathrm{m}^{2}\right)$. Similarly, compared with PHODA-0, PH-ODA-10 shows a $62.3 \%$ reduction in total heat release (THR) value. The low HRR and THR values further demonstrate the excellent flame-retarded performance of IFRs. The smoke released from combustion is deemed as an extremely important parameter for fire safety of epoxy resins. ${ }^{34}$

Table 2 Results of UL-94 and LOI tests for cured epoxy resin

\begin{tabular}{lllll}
\hline Samples & LOI $(\%)$ & $t_{1}+t_{2}(\mathrm{~s})$ & Dripping & UL-94 rating \\
\hline PH-ODA-0 & 23.5 & Last burning & Yes & No rating \\
PH-ODA-5 & 27.8 & Last burning & No & No rating \\
APP-10 & 29.1 & $85.8 \pm 9.5$ & No & No rating \\
PH-ODA-10 & 29.9 & $1.1 \pm 0.6$ & No & V-0 \\
PH-ODA-15 & 32.3 & $0.5 \pm 0.3$ & No & V-0
\end{tabular}

In Fig. 2c and d, compared to PH-ODA-0, the peak smoke production rate (pSPR) and total smoke production (TSP) values of PH-ODA-10 are decreased significantly by $83.1 \%$ and $68.3 \%$, demonstrating that the present IFRs are very beneficial to the smoke suppression of epoxy resins. Table $\mathrm{S} 1 \dagger$ lists the reported Schiff base structures for flame retardant epoxy resins. As seen, most of the reported Schiff base compounds contain P element, because they are usually synthesized from the reaction of Schiff base intermediates with DOPO or DPPA, which lead to the high molecular weights of these Schiff base compounds. In this work, the flame retardancy of PH-ODA is attributed to the good cross-linking charring ability of $\mathrm{C}=\mathrm{N}$ double bond (without introducing DOPO or DPPA), which shows a relatively lower molecular weight compared with the reported Schiff base compounds and thus reduces the steric hindrance of PH-ODA molecule. In addition, the four active phenolic groups make PH-ODA have good compatibility with epoxy resin.

\section{Char analysis}

Fig. $\mathrm{S} 2 \uparrow$ depicts the photos of the char residues after CCT. It is revealed that the char residue of PH-ODA-0 is fragile and only a little char remains due to its poor charring property. In contrast, the PH-ODA-10 possesses highly compact and intumescent char residues. The observation from SEM (Fig. S3†) shows that the char residue of PH-ODA-0 possesses the cracked and discontinuous char residue with many open holes. For the char residue of PH-ODA-10, many tunnels and a more homogeneous and continuous char residue is generated, which provides a protective barrier for isolating the gases and heat from the epoxy matrix.

FTIR technique is utilized for analyzing the chemical compositions of char residues after CCT. For char residues of both PH-ODA-0 and PH-ODA-10 in Fig. 3, the peak appeared at $3452 \mathrm{~cm}^{-1}(\mathrm{~N}-\mathrm{H}$ and $\mathrm{O}-\mathrm{H})$ indicate that amino- and hydroxylcontaining substances exist in PH-ODA-0 and PH-ODA-10 after combustion. The peak at $1631 \mathrm{~cm}^{-1}$ is assigned to the stretching vibrations of carbonized compounds. Meanwhile, for $\mathrm{PH}-$ ODA-10, several new absorption peaks appear, which are assigned to the phosphorus-containing bands: $1082 \mathrm{~cm}^{-1}(\mathrm{P}=$ O), $903 \mathrm{~cm}^{-1}$ (P-O-C) and $1235 \mathrm{~cm}^{-1}(\mathrm{P}-\mathrm{O}-\mathrm{C}){ }^{35}$ The FTIR results indicate that the nitrogen and phosphorus-containing substances are left after CCT, which jointly promote the formation of intumescent char during the combustion of epoxy matrix. XPS technique is also utilized for analyzing the element composition of the char residues, as listed in Table 4. The char residue of $\mathrm{PH}-\mathrm{ODA}-0$ is composed of $\mathrm{C}, \mathrm{O}$ and $\mathrm{N}$ elements and contains no $\mathrm{P}$ element. Meanwhile, the $\mathrm{P}$ content of the $\mathrm{PH}-$ ODA-10 char residue reaches $2.70 \mathrm{wt} \%$, indicating that the 

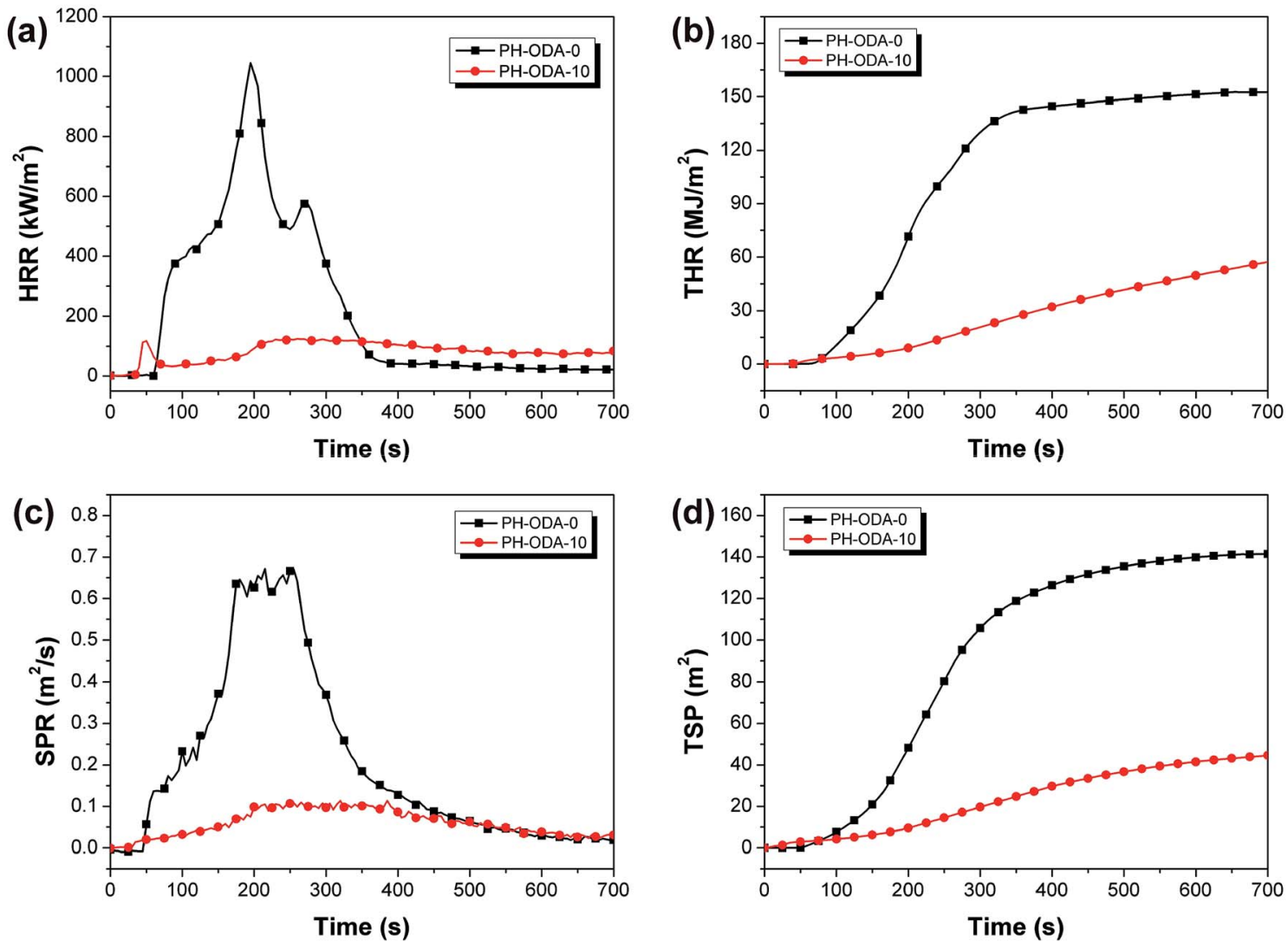

Fig. 2 (a) HRR, (b) THR, (c) SPR, and (d) TSP curves of PH-ODA-0 and PH-ODA-10.

phosphorus-containing substances generated from PH-ODA-10 play a very important role in forming the intumescent char, which agrees with the results of FT-IR.

Raman spectroscopy is applied for assessing the graphitization of char by using the ratio of the integrated intensities of $\mathrm{D}$ to $\mathrm{G}$ bands on Raman spectra. Fig. $\mathrm{S} 4 \uparrow$ illustrates the Raman spectra of char residues for PH-ODA-0 and PH-ODA-10, both of which show two distinctive bands i.e. $\mathrm{D}$ band $\left(1345 \mathrm{~cm}^{-1}\right)$ and $\mathrm{G}$ band $\left(1591 \mathrm{~cm}^{-1}\right)$. D band is related to the disordered carbon structure and $\mathrm{G}$ band is related to the stretching vibration of carbon atoms in the crystalline part of graphite layers. ${ }^{36}$ Thus, the ratio of integrated intensities $\left(I_{\mathrm{D}} / I_{\mathrm{G}}\right)$ is an indicator of the degree of graphitization. The $I_{\mathrm{D}} / I_{\mathrm{G}}$ values of PH-ODA-0 char (3.46) is much higher than that of PH-ODA-10 char (3.10). The lower $I_{\mathrm{D}} / I_{\mathrm{G}}$ value of PH-ODA-10 suggests that the char of $\mathrm{PH}$ ODA-10 possess higher degree of graphitization, indicating that the IFRs (PH-ODA/APP blends) are helpful to enhance the thermal stability and flame retardancy of epoxy composite. ${ }^{37}$

Table 3 Cone calorimetry data for cured epoxy resin

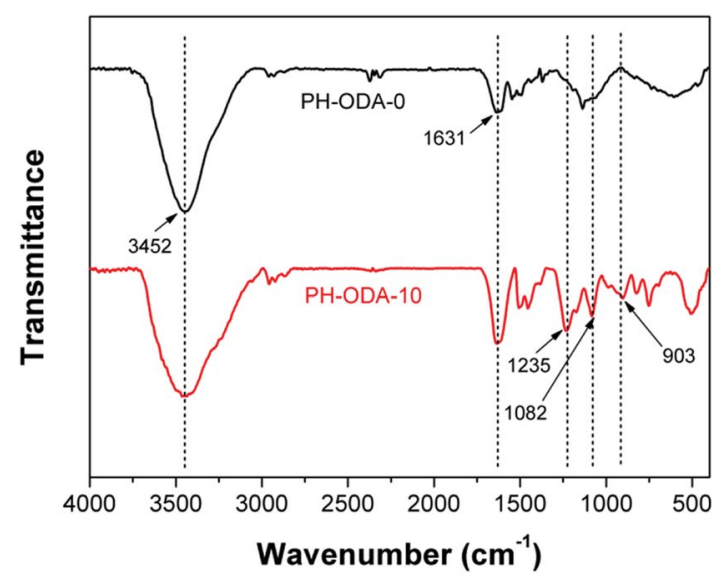

Fig. 3 FT-IR spectra of char residues after CCT.

\section{Thermogravimetric analysis}

Fig. 4 shows TGA curves of PH-ODA-0 and PH-ODA-10 $\left(\mathrm{N}_{2}\right.$ atmosphere). It is seen that PH-ODA-0 and PH-ODA-10 possess

\begin{tabular}{|c|c|c|c|c|c|}
\hline PH-ODA-0 & 50 & 1045.2 & 152.3 & 0.65 & 141.2 \\
\hline
\end{tabular}


Table 4 XPS analysis of char residues after CCT

\begin{tabular}{lllll}
\hline Samples & $\mathrm{C}(\mathrm{wt} \%)$ & $\mathrm{O}(\mathrm{wt} \%)$ & $\mathrm{N}(\mathrm{wt} \%)$ & $\mathrm{P}(\mathrm{wt} \%)$ \\
\hline PH-ODA-0 & 80.87 & 14.92 & 4.21 & 0 \\
PH-ODA-10 & 85.75 & 9.82 & 1.73 & 2.70
\end{tabular}

the single weight loss process. In Fig. 4a, PH-ODA-10 starts to degrade at a lower temperature $\left(T_{\mathrm{d} 5 \%}=350.7^{\circ} \mathrm{C}\right)$ than PH-ODA$0\left(388.2^{\circ} \mathrm{C}\right)$, and degrades more quickly in the initial degradation stage $\left(350-420^{\circ} \mathrm{C}\right)$, indicating that the early degradation of IFRs facilitates the degradation of epoxy resin, which agrees with the TTI results. When the temperature exceeds $420{ }^{\circ} \mathrm{C}$, the residue of $\mathrm{PH}-\mathrm{ODA}-10$ is larger than that of $\mathrm{PH}-\mathrm{ODA}-0$, suggesting that the early degraded IFRs stabilizes the residue at higher temperature. Moreover, the residue $\left(700^{\circ} \mathrm{C}\right)$ of PH-ODA10 is increased to $27.1 \%$ from $14.8 \%$. The results are in accordance with the SEM results, suggesting that IFRs promote the char forming during the thermal degradation process and enhance cross-linking reactions in epoxy composites at higher temperature range, improving the thermal stability and char residue of epoxy composites.

The interaction between PH-ODA and APP is verified by the comparison of the calculated and experimental results of $\mathrm{PH}$ ODA/APP mixture during the thermal degradation (Fig. 4b). The calculated curve is achieved based on the combination of the two separate TGA curves of PH-ODA and APP.$^{38}$ It can be seen that the experimental curve is above the calculated curve after $680{ }^{\circ} \mathrm{C}$, suggesting that the reaction between PH-ODA and APP improves the thermal stability and charring properties. In addition, the residue of the experimental curve at $800{ }^{\circ} \mathrm{C}(30.0 \%)$ is higher than the calculated residue $(23.7 \%)$. The above results further verify the synergistic effect in the IFRs system, which promote the char formation of the epoxy composite.

\section{TG-FTIR analysis of gaseous phase}

TG-FTIR technique is used to investigate the flame-retardant mechanism by detecting the pyrolysis gas generated from the degradation of PH-ODA-0 and PH-ODA-10. According to the TGA result, both PH-ODA-0 and PH-ODA-10 possess the single degradation process. The FTIR spectra at the initial (corresponding to $350{ }^{\circ} \mathrm{C}$ for PH-ODA-10 and $390{ }^{\circ} \mathrm{C}$ for PH-ODA-0) and maximum (corresponding to $370{ }^{\circ} \mathrm{C}$ for PH-ODA-10 and $405{ }^{\circ} \mathrm{C}$ for $\mathrm{PH}-\mathrm{ODA}-0$ ) degradation temperatures are illustrated in Fig. 5. The pyrolysis products of both PH-ODA-0 and PH-ODA10 are analyzed as follow: $3564 \mathrm{~cm}^{-1}$ (phenol derivatives), 3037 and $2960 \mathrm{~cm}^{-1}$ (aliphatic hydrocarbons), 2343 and $2302 \mathrm{~cm}^{-1}$ $\left(\mathrm{CO}_{2}\right), 1598,1503$, and $1332 \mathrm{~cm}^{-1}$ (aromatic substances), 1251 and $1170 \mathrm{~cm}^{-1}$ (ester and ether compounds). ${ }^{35,39}$ Compared with PH-ODA-0, two new peaks occur in the spectrum of $\mathrm{PH}-$ ODA-10, which are assigned to the $\mathrm{NH}_{3}\left(931\right.$ and $\left.960 \mathrm{~cm}^{-1}\right) .{ }^{40}$ The results confirm that the nitrogen-containing gaseous products from the IFRs in PH-ODA-10 appear in the pyrolysis gas, which has a positive effect on the fire resistance of epoxy resin in vapor phase.

\section{FTIR analysis of condensed-phase}

In order to investigate the flame-retardant mechanism of IFRs (PH-ODA/APP mixture) in condense phase, the residues of $\mathrm{PH}$ ODA/APP mixture (at different temperature) were detected by FTIR (Fig. 6). As seen, compared with the spectrum at $25^{\circ} \mathrm{C}$, the peaks at $1585 \mathrm{~cm}^{-1}$ and $1437 \mathrm{~cm}^{-1}\left(\mathrm{NH}_{4}{ }^{+}\right.$stretching) disappear after $300{ }^{\circ} \mathrm{C}$, which is due to the removal of $\mathrm{NH}_{3}$ and agrees with the TG-FTIR results. In addition, the peak of PH-ODA at $3335 \mathrm{~cm}^{-1}$ (-OH stretching) disappear and peaks at 1253 and $914 \mathrm{~cm}^{-1}$ (P-O-C stretching) appear after $300{ }^{\circ} \mathrm{C}$, which is attributed to the dehydration reaction of PH-ODA with polyphosphoric acid. Besides, the peak at $1001 \mathrm{~cm}^{-1}(\mathrm{P}=\mathrm{O}$ stretching) is found over the whole temperature scope. The above results show that $\mathrm{P}-\mathrm{O}-\mathrm{C}$ and $\mathrm{P}=\mathrm{O}$ promote the formation of char layer during the combustion of epoxy matrix. The peak appeared at $1650 \mathrm{~cm}^{-1}\left(-\mathrm{C}=\mathrm{C}\right.$ - stretching) after $300{ }^{\circ} \mathrm{C}$ indicates the generation of carbonized substances. Moreover, the peak of PH-ODA at $1487 \mathrm{~cm}^{-1}(-\mathrm{C}=\mathrm{N}$ - stretching) disappear and peak at $1401 \mathrm{~cm}^{-1}\left(-\mathrm{C}-\mathrm{N}\right.$ - stretching) appear at $300^{\circ} \mathrm{C}$, indicating the formation of amine nitrogen $(-\mathrm{C}-\mathrm{N}-)$. According to the literature, ${ }^{26}$ the amine nitrogen $(-\mathrm{C}-\mathrm{N}-)$ converts to nitrogen-containing hexatomic ring after further increasing the temperature. The above results indicate that the reaction of APP
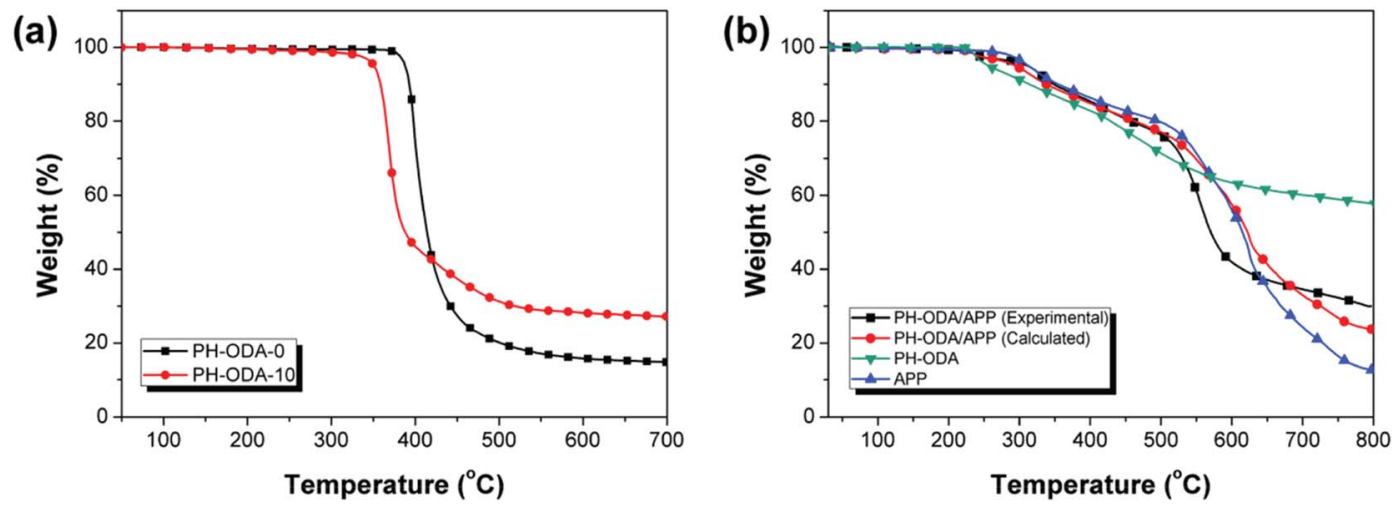

Fig. 4 TGA curves of (a) PH-ODA-O and PH-ODA-10 in $\mathrm{N}_{2}$ and (b) experimental and calculated PH-ODA/APP blends in $\mathrm{N}_{2}$. 

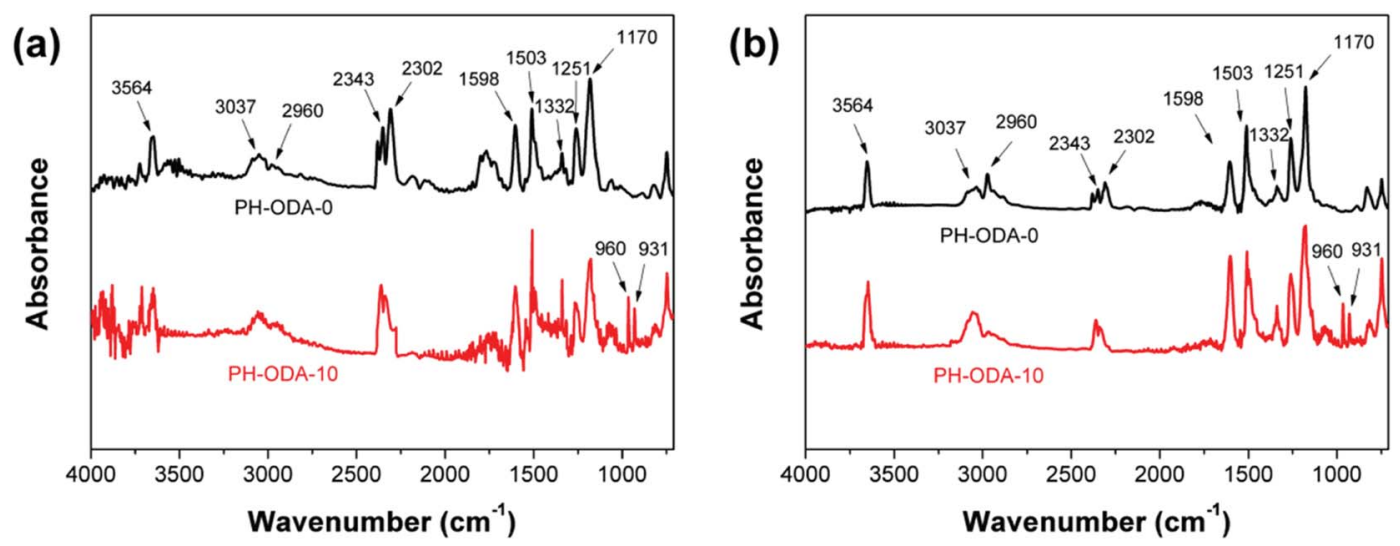

Fig. 5 The FTIR spectra of pyrolysis products of PH-ODA-10 and PH-ODA-0 at (a) the initial and (b) maximum degradation temperatures.

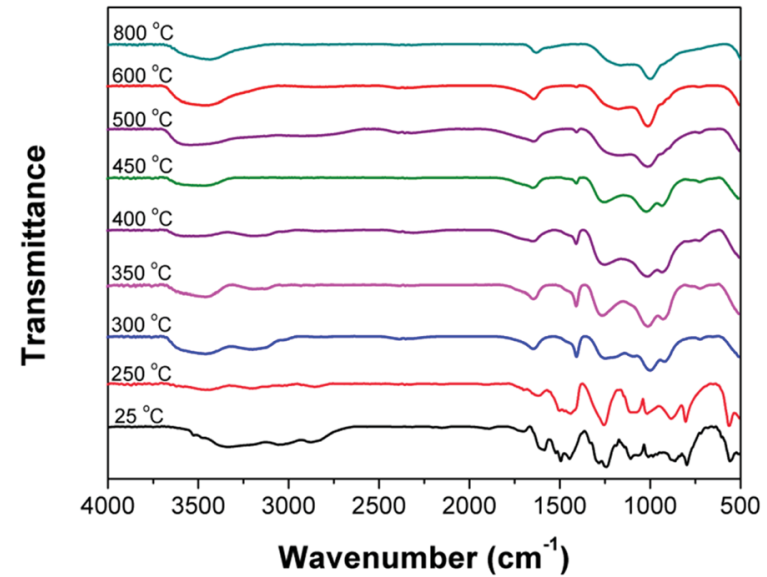

Fig. 6 FTIR spectra of the residues of IFRs (PH-ODA/APP blends) heated at different temperatures.

with PH-ODA and the self-crosslinking of $-\mathrm{C}=\mathrm{N}$ - in PH-ODA jointly promote the char forming of the stable char layer.

\section{Thermomechanical and mechanical properties}

DMA is applied for the evaluation of thermomechanical properties of cured epoxy thermosets (Fig. 7 and Table 5). PH-ODA-
10 shows higher storage modulus $\left(E^{\prime}\right)$ value $(3.29 \mathrm{GPa})$ than $\mathrm{PH}^{-}$ ODA-0 (2.97 GPa) at room temperature, suggesting that the rigid structure of IFRs enhances the stiffness of epoxy thermosets. Besides, PH-ODA-10 possesses a $T_{\mathrm{g}}$ of $159.5^{\circ} \mathrm{C}$, which is slightly lower than PH-ODA-0 $\left(162.2^{\circ} \mathrm{C}\right)$. Although the four phenolic groups in PH-ODA can react with the epoxy groups, which makes the PH-ODA have better compatibility with epoxy resin. However, the poor interfacial adhesion between the APP (main component in IFRs) and epoxy matrix may lead to the decrease in $T_{\mathrm{g}}$, which is in accordance with the results in the literature. ${ }^{41}$

The mechanical properties of PH-ODA- 0 and PH-ODA- 10 are further evaluated (see Table 6). Compared to PH-ODA-0, the tensile and flexural strengths of PH-ODA-10 are only decreased by $5.1 \%$ and $4.2 \%$, respectively. These differences are mainly

Table 5 Key parameters collected from DMA for $\mathrm{PH}-\mathrm{ODA}-\mathrm{O}$ and $\mathrm{PH}$ ODA-10

\begin{tabular}{llll}
\hline & $\begin{array}{l}E^{\prime} \\
\text { at } 30{ }^{\circ} \mathrm{C}(\mathrm{GPa})\end{array}$ & $T_{\mathrm{g}}\left({ }^{\circ} \mathrm{C}\right)$ & $\nu_{\mathrm{e}}\left(10^{3} \mathrm{~mol} \mathrm{~m}^{-3}\right)$ \\
\hline PH-ODA-0 & 2.97 & 162.1 & 5.04 \\
PH-ODA-10 & 3.29 & 153.3 & 4.95
\end{tabular}
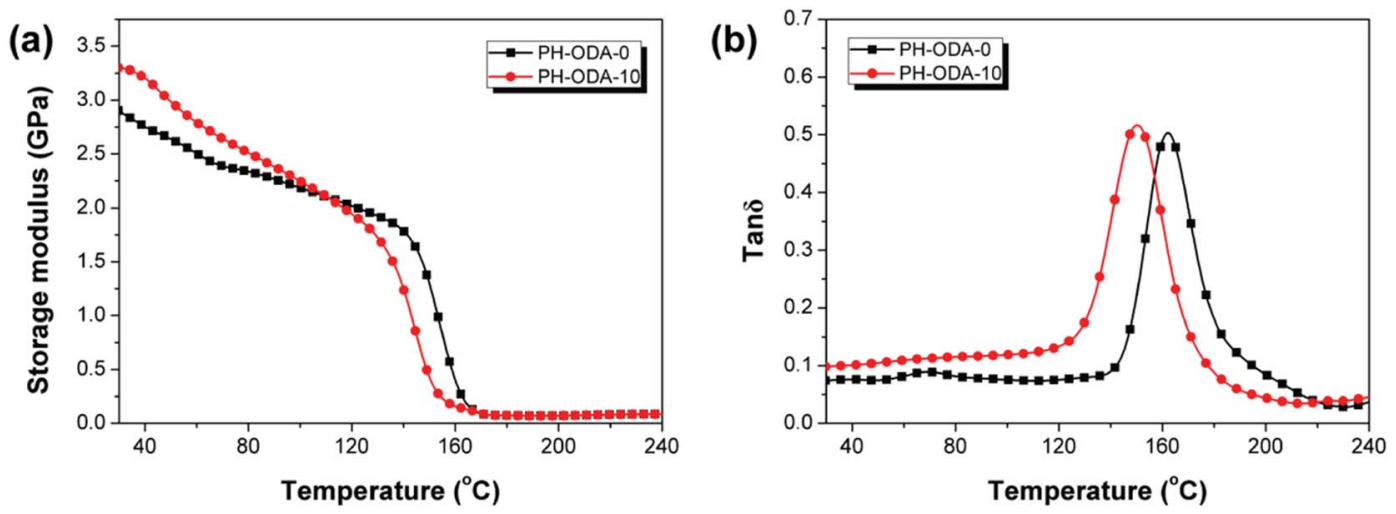

Fig. 7 (a) Storage modulus ( $\left.E^{\prime}\right)$ and (b) tan $\delta$ curves for PH-ODA-O and PH-ODA-10. 
Table 6 Tensile and flexural properties of cured epoxy resin

\begin{tabular}{|c|c|c|c|c|}
\hline Samples & Tensile modulus (GPa) & $\begin{array}{l}\text { Tensile strength } \\
\text { (MPa) }\end{array}$ & Flexural modulus (GPa) & $\begin{array}{l}\text { Flexural strength } \\
(\mathrm{MPa})\end{array}$ \\
\hline PH-ODA-0 & $3.32 \pm 0.23$ & $72.8 \pm 1.0$ & $2.71 \pm 0.11$ & $108.2 \pm 5.0$ \\
\hline PH-ODA-10 & $3.42 \pm 0.12$ & $69.1 \pm 0.9$ & $2.92 \pm 0.23$ & $103.7 \pm 1.6$ \\
\hline
\end{tabular}

originated from the lower cross-link density of PH-ODA-10. Meanwhile, the tensile and flexural moduli of PH-ODA-10 are respectively increased by $3.0 \%$ and $7.7 \%$, which is mainly a result of the more rigid structures (Schiff base and aromatic structure) in PH-ODA-10.

\section{Conclusions}

In summary, a novel bio-based Schiff base compound PH-ODA was successfully synthesized and acted as a carbonization agent of IFRs for fire-safe epoxy resins. Only $10 \mathrm{wt} \%$ IFRs $(2.5 \mathrm{wt} \% \mathrm{PH}-$ ODA and $7.5 \mathrm{wt} \%$ APP) were sufficient to pass UL-94 V-0 rating. The PHRR and THR of PH-ODA-10 were respectively $88.1 \%$ and $62.3 \%$ lower than those of pure epoxy resins. Similarly, the pSPR and TSP values of PH-ODA-10 were decreased by $83.1 \%$ and $68.3 \%$. The enhancement of fire-safety performance was attributed to that $\mathrm{PH}-\mathrm{ODA} / \mathrm{APP}$ promoted the formation of more compact intumescent char structure. This work provides an effective strategy to prepare bio-based carbonization agent of IFRs for epoxy thermosets.

\section{Conflicts of interest}

The authors declare that there are no conflicts of interest.

\section{Acknowledgements}

We gratefully acknowledge support from the National Natural Science Foundation of China (No. 51404040 and 51573054) and the Opening Project of Key Laboratory of Polymer Processing Engineering (South China University of Technology), Ministry of Education, China (No. KFKT1702).

\section{References}

1 H. Jiang, L. Sun, Y. Zhang, Q. Liu, C. Ru, W. Zhang and C. Zhao, Polym. Degrad. Stab., 2019, 160, 45-52.

2 T. Liu, X. Guo, W. Liu, C. Hao, L. Wang, W. C. Hiscox, C. Liu, C. Jin, J. Xin and J. Zhang, Green Chem., 2017, 19, 4364-4372.

3 S. Wang, S. Ma, Q. Li, X. Xu, B. Wang, W. Yuan, S. Zhou, S. You and J. Zhu, Green Chem., 2019, 21, 1484-1497.

4 Y. Shi and G. Wang, Prog. Org. Coat., 2016, 97, 1-9.

5 J. Zhang, Q. Kong and D.-Y. Wang, J. Mater. Chem. A, 2018, 6, 6376-6386.

6 C. Ma, S. Qiu, B. Yu, J. Wang, C. Wang, W. Zeng and Y. Hu, Chem. Eng. J., 2017, 322, 618-631.

7 Y. Shi, B. Yu, Y. Zheng, J. Yang, Z. Duan and Y. Hu, J. Colloid Interface Sci., 2018, 521, 160-171.
8 X. Wang, S. Zhou, W.-W. Guo, P.-L. Wang, W. Xing, L. Song and Y. Hu, ACS Sustainable Chem. Eng., 2017, 5, 3409-3416.

9 S. Yang, J. Wang, S. Huo, M. Wang and L. Cheng, Ind. Eng. Chem. Res., 2015, 54, 7777-7786.

10 S. Qiu, W. Xing, X. Feng, B. Yu, X. Mu, R. K. K. Yuen and Y. Hu, Chem. Eng. J., 2017, 309, 802-814.

11 E. N. Kalali, X. Wang and D.-Y. Wang, Ind. Eng. Chem. Res., 2016, 55, 6634-6642.

12 S. Yang, J. Wang, S. Huo, L. Cheng and M. Wang, Polym. Degrad. Stab., 2015, 119, 251-259.

13 C. Ma, B. Yu, N. Hong, Y. Pan, W. Hu and Y. Hu, Ind. Eng. Chem. Res., 2016, 55, 10868-10879.

14 X. Su, Y. Yi, J. Tao, H. Qi and D. Li, Polym. Degrad. Stab., 2014, 105, 12-20.

15 L. Zhou, Y. Ju, F. Liao, Y. Yang and X. Wang, Fire Mater., 2016, 40, 129-140.

16 M. Chen, M. Tang, Y. Ma, X. Chen, J. Qin, W. He and Z. Zhang, Polym. Eng. Sci., 2015, 55, 1355-1360.

17 M. Maqsood and G. Seide, Polymers, 2019, 11, 48.

18 M. Maqsood, F. Langensiepen and G. Seide, Molecules, 2019, 24, 1513.

19 X. Ye, Y. Wang, Z. Zhao and H. Yan, Polym. Degrad. Stab., 2017, 142, 29-41.

20 C. Huayao, L. Yueshun, Z. Hongjun, Z. Xinhua, G. Sheng and X. Hua, RSC Adv., 2016, 6, 114714-114721.

21 D. Zhao, H. Yu, S. Mei, K. Pan and J. Deng, Ind. Eng. Chem. Res., 2019, 58, 1105-1113.

22 N. Ye, X. Wang, Q. Liu and X. Hu, Anal. Chim. Acta, 2018, 1028, 113-120.

23 A. Naghipour and A. Fakhri, Catal. Commun., 2016, 73, 3945.

24 A.-H. Yang, C. Deng, H. Chen, Y.-X. Wei and Y.-Z. Wang, Polym. Degrad. Stab., 2017, 144, 70-82.

25 D. Zhao, J. Wang, X.-L. Wang and Y.-Z. Wang, Chem. Eng. J., 2018, 344, 419-430.

26 J.-N. Wu, L. Chen, T. Fu, H.-B. Zhao, D.-M. Guo, X.-L. Wang and Y.-Z. Wang, Chem. Eng. J., 2018, 336, 622-632.

27 X. Shi, Y. Ju, M. Zhang and X. Wang, Fire Mater., 2018, 42, 190-197.

28 L. Costes, F. Laoutid, S. Brohez and P. Dubois, Mater. Sci. Eng., $R, 2017,117,1-25$.

29 L. Yang, L. Wu, X. Yao, S. Zhao, J. Wang, S. Li and W. Ding, Microbiol. Res., 2018, 215, 15-21.

30 S. Li, Y. Yu, J. Chen, B. Guo, L. Yang and W. Ding, Molecules, 2016, 21, 754.

31 X. Liu, X. Gu, J. Sun and S. Zhang, Carbohydr. Polym., 2017, 167, 356-363. 
32 S. Tang, V. Wachtendorf, P. Klack, L. Qian, Y. Dong and B. Schartel, RSC Adv., 2017, 7, 720-728.

33 E. N. Kalali, X. Wang and D.-Y. Wang, J. Mater. Chem. A, 2015, 3, 6819-6826.

34 X.-L. Li, F.-H. Zhang, R.-K. Jian, Y.-F. Ai, J.-L. Ma, G.-J. Hui and D.-Y. Wang, Composites, Part B, 2019, 107200.

35 C. Dong, A. Wirasaputra, Q. Luo, S. Liu, Y. Yuan, J. Zhao and Y. Fu, Materials, 2016, 9, 1008.

36 D. Tiwari, H. Bhunia and P. K. Bajpai, Appl. Surf. Sci., 2018, 439, 760-771.
37 C. S. R. Gangireddy, X. Wang, Y. Kan, L. Song and Y. Hu, Polym. Int., 2019, 68, 936-945.

38 C. Chen, X. Gu, X. Jin, J. Sun and S. Zhang, Carbohydr. Polym., 2017, 157, 1586-1593.

39 Q. Luo, Y. Yuan, C. Dong, H. Huang, S. Liu and J. Zhao, Ind. Eng. Chem. Res., 2016, 55, 10880-10888.

40 V. Realinho, L. Haurie, J. Formosa and J. I. Velasco, Polym. Degrad. Stab., 2018, 155, 208-219.

41 Q. Tang, B. Wang, Y. Shi, L. Song and Y. Hu, Ind. Eng. Chem. Res., 2013, 52, 5640-5647. 